\title{
Learning outcomes of a group behavioral modification program to prevent urinary incontinence
}

Received: 11 August 2004 / Accepted: 19 December 2004/Published online: 15 March 2005

(c) International Urogynecology Journal 2005

\begin{abstract}
This study describes acquisition of knowledge and motor skill in bladder training (BT) and pelvic floor muscle training (PFMT) and adherence following a behavioral modification program (BMP). Essentially continent $(0-5$ episodes in past year) community-dwelling older women $(n=359)$ were randomized to treatment $(n=164)$, a 2-h group education session supplemented by one brief individualized session of approximately $10 \mathrm{~min}$, or control $(n=195)$, no instruction, and followed for 12 months. Knowledge, motor skill, and adherence to the BMP were documented. Changes in pelvic muscle function and voiding interval were used to validate self-reported adherence. Following group instruction, mean BT and PFMT knowledge was 90 and $86 \%$, respectively; $68 \%$ demonstrated correct PFMT technique without additional instruction, 29\% required brief instruction, and 3\% were unable to learn PFMT technique. Adherence ranged from 63 to $82 \%$ for PFMT and 58 to $67 \%$ for BT. Group instruction supplemented
\end{abstract}

\footnotetext{
C. M. Sampselle $(\varangle)$

Obstetrics and Gynecology, School of Nursing,

University of Michigan, 400 North Ingalls,

Ann Arbor, MI 48109-0482, USA

E-mail: csampsll@umich.edu

Tel.: + 1-734-7649555

Fax: + 1-734-6151666

K. L. Messer · S. H. Hines

School of Nursing, University of Michigan,

400 North Ingalls, Ann Arbor, MI 48109-0482, USA

J. S. Seng

Department of Obstetrics and Gynecology,

School of Nursing, University of Michigan,

400 North Ingalls, Ann Arbor, MI 48109-0482, USA

T. E. Raghunathan

Institute for Social Research, University of Michigan, 400 North Ingalls, Ann Arbor, MI 48109-0482, USA

A. C. Diokno

Department of Urology, William Beaumont Hospital, Royal Oak, MI, USA
}

with brief individual instruction as needed is an effective teaching method for BT and PFMT.

Keywords Behavioral modification - Bladder training Group instruction · Pelvic floor muscle training ·

Prevention · Urinary incontinence

\section{Introduction}

It is widely accepted that behavioral intervention, specifically bladder training (BT) and pelvic floor muscle training (PFMT), are efficacious first-line therapies for women with urinary incontinence (UI) $[1,2]$. Although the efficacy of PFMT for prevention of UI has been shown with pregnant and postpartum women [16-18], our recently reported randomized controlled trial (RCT) was the first to our knowledge to demonstrate the preventive capacity of combined PFMT and BT in older women [3]. The rationale for that test of prevention was rooted in the expectation that these efficacious behavioral therapies would also decrease women's risk of developing UI.

In current practice, behavioral therapies are typically delivered one-on-one $[4,5]$. The literature about group education for BT and PFMT is limited, but two studies testing group versus no instruction of PFMT demonstrated decreased UI [6, 7]. Neither study reported knowledge or motor skill outcomes. We developed an education prevention program entitled the behavioral modification program (BMP) to teach the rationales, concepts, and skills of BT and PFMT to groups of women, first presenting a 2-h group session and following that with a single brief individualized session.

The aims of the study reported here were to describe BMP participants' acquisition of BT and PFMT knowledge and motor skill immediately following group instruction and to document adherence over 1 year. We addressed three research questions: 
1. What is the knowledge level following group instruction in BT and PFMT?

2. What proportions of women demonstrate correct motor skill in performance of pelvic muscle contraction following group instruction in PFMT?

3. What is the level of self-reported adherence following group instruction in BT and PFMT and a single individualized one-on-one session?

\section{Patients and methods}

\section{Participants}

Community-dwelling women 55-80 years of age were assessed for continence status and, if they met the criteria for continence (either zero incontinence or no more than five episodes of incontinence in the past year), were enrolled in the study. Participants were recruited in two ways, i.e., phased mass mailings into targeted community zip codes and referral from the Human Subject Core of the Older Americans Independence Center (OAIC) at the University of Michigan. Respondents to the invitation to participate were initially screened by telephone, randomized if they met the initial telephone inclusion criteria, and scheduled for a clinical examination where final screening occurred if they were in the control group. Women in the treatment group were scheduled for the group education segment of the BMP, after which they received an appointment for a clinical examination 2-4 weeks later, where final screening occurred and individualized instruction was provided as needed. The individualized instruction addressed deficient knowledge of BT and PFMT and incorrect pelvic

Table 1 Inclusion criteria

Telephone criteria

$1 \quad$ Female 55 years through 80 years of age

2 Postmenopausal defined as no menstrual cycle for the past 12 months except those who are on hormone replacement therapy

3 Continent of urine by the criteria used in our previous study's definition of no UI ever except during pregnancy and immediate postpartum period, or urine loss of no more than 1-5 days during the last 12 months, and no previous or present UI treatment with pharmacologic agents, behavioral techniques, or surgical therapy

$4 \quad$ No neurologic disease

$5 \quad$ No difficulties with activities of daily living

Clinic criteria

1 Self-report UI questionnaire showing no UI or UI episodes of 5 days or less in the last 12 months

2 Mini-mental status score of 24 or higher

3 Negative paper towel cough stress test with bladder volume at least $150 \mathrm{ml}$

$4 \quad$ No grade 4 uterine prolapse (prolapsing beyond the vaginal introitus) on pelvic examination

5 No introital stenosis precluding digital examination

6 Ability to voluntarily contract pelvic muscles muscle contraction technique. Inclusion criteria for telephone and clinic screening are delineated in Table 1. A key component of the clinic screening battery was the paper towel test [8], an objective assessment of UI that confirmed continence during deep coughing with a full bladder and demonstrated ability to voluntarily contract the pelvic floor muscles.

From the phased mailings (approximately 47,400) and the UM OAIC program (179), 639 volunteers qualified at the telephone-screening interview (569 mailing, 43 UM OAIC) of which $480(75 \%)$ consented and were randomized to the treatment group $(n=238)$ or control group $(n=242)$. Nine from control and 21 from treatment elected not to participate, leaving 217 treatment and 233 control participants prior to the clinic visit. Of the 450 participants who received the clinic screening, 38 control and 53 treatment participants (91) were eliminated. Sixty-eight failed the eligibility criteria and 23 refused or had missing data, leaving 164 treatment and 195 control participants.

Of the 359 participants who passed the screening tests and completed all baseline measures, 318 completed the study with data on outcome measures. The full year follow-up period was not completed by 18 of $195(9 \%)$ in the control group and 23 of $164(14 \%)$ in the treatment group. Reasons for drop out included loss to follow-up, relocation, and declining health of self or family member.

Table 2 Sociodemographic characteristics of participants by group Sociodemographic characteristics of study participants at baseline, $n=359^{\mathrm{a}}$

\begin{tabular}{|c|c|c|}
\hline Variable and description & $\begin{array}{l}\text { Control group } \\
n=195^{\mathrm{b}} \\
\%\end{array}$ & $\begin{array}{l}\text { Treatment group } \\
n=164^{\mathrm{b}} \\
\%\end{array}$ \\
\hline \multicolumn{3}{|l|}{ Race } \\
\hline White & 81 & 84 \\
\hline \multicolumn{3}{|l|}{ Education } \\
\hline Greater than high school & 78 & 82 \\
\hline \multicolumn{3}{|l|}{ Family income } \\
\hline Less than $20 \mathrm{~K}$ & 12 & 14 \\
\hline $20-50 \mathrm{~K}$ & 43 & 31 \\
\hline Greater than $50 \mathrm{~K}$ & 45 & 55 \\
\hline \multicolumn{3}{|l|}{ Baseline health } \\
\hline Excellent & 18 & 23 \\
\hline Very good & 49 & 49 \\
\hline Good & 32 & 27 \\
\hline Fair & 1 & 1 \\
\hline \multirow[t]{2}{*}{ One or more births } & 88 & 90 \\
\hline & Mean/SD & Mean/SD \\
\hline $\begin{array}{l}\text { Mini-mental at } \\
\text { entrance }(1-32)^{\mathrm{c}}\end{array}$ & $31.3 / 1.3$ & $31.1 / 1.3$ \\
\hline $\begin{array}{l}\text { Mini-mental } \\
\text { at exit }(1-32)^{\mathrm{c}}\end{array}$ & $31.4 / 1.2$ & $31.4 / 1.1$ \\
\hline
\end{tabular}

${ }^{\text {a }} n$ may be different across variables due to missing values ${ }^{\mathrm{b}}$ None of the differences between groups are significant

${ }^{\mathrm{c}}$ Modified mini-mental score-two additional items; allowed possible score of 32 
Baseline sociodemographic, medical history, and incontinence characteristics of the treatment and control groups are presented in Table 2. There were no significant differences between the two groups at baseline.

\section{Procedures}

The participants randomized to the treatment group received a 2-h group education session, the BMP. Those in the control group received no such education. Both groups were examined by a nurse with specialized training in continence care to confirm eligibility. Those in the BMP group had this examination 2-4 weeks after the group session and received additional individualized instruction in BT and PFMT as needed.

The group education session of the BMP was composed of two segments, with slides and handouts for each. It was taught in groups ranging from 5 to 25 participants. The first hour, presented by the urologist investigator, covered anatomy and physiology of the lower urinary tract and pelvis, daily fluid intake requirement, voiding frequency and bladder capacity, types and causes of UI, and life impact of UI. The second hour, presented by the nurse investigator, included concepts of self-care, identification of personal voiding interval, instruction in BT, instruction and practice in locating and exercising pelvic floor muscles, prescription for PFMT, and strategies to incorporate BT and PFMT into one's daily life. Each segment was followed by a question and answer period. The learning experiences selected for this group session were guided by four sources of information originally posited by Bandura [9] to increase self-efficacy: verbal persuasion, emotional arousal, vicarious experience, and performance accomplishments. These sources were implemented in the group session as follows: (1) Verbal persuasion suggested that women could successfully implement selfcare practices to prevent UI. The evidence base for the efficacy of BT and PFMT was discussed. (2) Emotional arousal was designed to increase women's awareness of the decreased quality of life associated with UI. Samples of products for the management of UI were examined and participants were asked how their lives would change should they develop UI. (3) Vicarious experience was provided with case examples of other women who successfully implemented the behavioral strategies. During group discussion, participants shared how the development of UI might affect them personally and planned how they could incorporate BT and PFMT into their personal schedules. Each received a magnet to display as a personal reminder to sustain the habits. (4) Performance accomplishment information stems from personal mastery experiences. During the group session, data from participants' 3-day voiding diaries were used to calculate each woman's current voiding interval to provide an individualized starting point for BT, and a practice session of PFMT was conducted using the audiotape that was presented to each "graduate" of the group session. The audiotape progressively advanced pelvic muscle repetitions over a 3-week period or longer as needed to the recommended frequency of 50 per day. Discussions were also guided by the inoculation against setbacks recommended by Vinokur et al. [10-12] as a means of sustaining self-efficacy. Applying the inoculation concept in the BMP, women were alerted to the likelihood that they would occasionally forget or deliberately omit BT and PFMT. Strategies to address such lapses and to resume the BMP were discussed.

Within 2-4 weeks after the group session, treatment group participants were seen by the nurse specialist in UI for the purpose of evaluating each woman's understanding of BT and PFMT. The correctness of pelvic floor muscle contraction technique (motor skill) was assessed during a vaginal examination where the woman's ability to contract the pelvic floor up and in without exerting a bearing down effort was verified. For women who demonstrated adequate knowledge of BT and PFMT and who were able to perform a correct pelvic floor muscle contraction, no further training was provided. When indicated, the nurse specialist corrected misunderstandings about BT and PFMT and gave further individualized instruction (accomplished in approximately $10 \mathrm{~min}$ ) to improve pelvic floor muscle contraction technique.

Women in the control group were not asked questions about PFMT or BT because our inclusion criteria (Table 1) required that women have no previous or present UI treatment with behavioral techniques. To specifically ask women in the control group about BT and PFMT could heighten their awareness of these techniques and potentially contaminate the control condition.

Both the treatment and control groups were followed for a year after the baseline data point. In addition to the baseline and 12-month exit point questionnaires, 3-day voiding diaries, and clinical evaluations, participants completed questionnaires and 3-day voiding diaries at 3month intervals throughout the year of the study. Upon collection of the exit data, control participants were invited to attend a group BMP session and were extended an opportunity for individualized instruction with a nurse specialist.

Knowledge acquisition, motor skill, and adherence were assessed in members of the treatment group. BT and PFMT knowledge was evaluated twice, immediately after the group session and prior to the clinical exam, by multiple-choice questions. The three questions asked about BT knowledge and the five about PFMT knowledge are presented in Table 3 . Using the period of 2-4 weeks from the completion of the BMP group session to questions answered prior to exam, test-retest reliability was calculated. The percent agreement across the eight items ranged from 60 to $100 \%$ and averaged $87 \%$ for the BT questions and $82 \%$ for the PFMT questions.

Correct PFMT technique was assessed by examination at 2-4 weeks post group instruction. To be scored 
Table 3 Multiple-choice questions about BT and PFMT (*indicates correct answer)

With regard to bladder training, what is the normal or target time you are aiming for between times of bladder emptying?

$1 \quad 2.5-3 \mathrm{~h}$ between each emptying

2 3.5-4 h between each emptying*

3 4.5-5 h between each emptying

Say you are on a 2 -h voiding schedule. You are due to void

at 9:30 a.m., but you have to empty earlier and void at 9:10 a.m.

When is the next time you should try to empty your bladder?

1 9:30 a.m.*

2 10:30 a.m.

3 11:10 a.m.

4 11:30 a.m.

How should you schedule voiding after your bedtime?

1 Try to get up for the scheduled times through the night

2 Just go to the bathroom as necessary, the schedule is only kept during waking hours*

3 Get up once about halfway through the night

Pelvic muscle exercise is intended to increase the strength of:

1 Muscles that support the bladder and urethra*

2 Thigh muscles

3 Abdominal muscles

The recommended position for LEARNING to do pelvic muscle exercise is:

1 Standing comfortably

2 Sitting in a chair

3 Lying down*

How many sets (exercise sessions) are recommended per day?

11

$22^{*}$

33

When you reach the highest level of pelvic muscle exercise,

how many contractions should you be doing in each set?

13 fast, 10 slow

25 fast, 20 slow*

310 fast, 25 slow

How long should you rest between each pelvic muscle contraction?

1 You should not rest, but do them as fast as you can

2 Rest at least $5 \mathrm{~s}$ between contractions

3 Rest at least $10 \mathrm{~s}$ between contractions*

as having mastered the desired technique (and requiring no further instruction), three characteristics of the contraction were confirmed: pelvic floor contracted up and in, pressure was exerted on the examining fingers, and bearing down effort was absent. The digital measure [13], a measure that has demonstrated capacity to selectively depict pelvic floor muscle activity [14], was used to quantify pelvic muscle strength. Contraction pressure was scored as: $1=$ none, $2=$ flick at one or more points on the examining fingers, but not completely around, 3 =full pressure completely around, and $4=$ compression, with possible overriding of the examining fingers. Displacement was scored as: $1=$ none, $2=$ base to mid-finger lifting, $3=$ total lifting of fingers to vigorous drawing up and in. Control group measures were obtained by instructing participants to squeeze or contract muscles in the vagina around the examiner's fingers, but no specific instructions in PFMT were given to control participants. Baseline measures were compared with the 12-month exit measures. Voiding frequency was assessed with a 3-day voiding diary developed by Wyman et al. [15] at baseline and compared at the 12-month exit point.
The PFMT adherence was determined by women's self-report of PFMT practice on the 3-, 6-, 9-, and 12month questionnaires as elicited by the question, "over the past 3 months, how often have you done the pelvic muscle exercises? (never, less than once a week, about once a week, 2 or 3 times a week, at least once a day, twice on most days, twice each day)." The BT adherence was determined by self-report on the 3-, 6-, 9-, and 12month questionnaires as elicited by the question, "during the past 3 months did you actually use BT? (yes, no)."

Descriptive statistics were calculated to determine knowledge, motor skill, and self-reported adherence. To test the hypotheses, two-sample $t$-tests were used to compare the means of continuous outcome variables for the two groups and two-sample binomial tests were used for dichotomous outcome variables.

\section{Results}

At 2-4 weeks following group education, knowledge about BT was assessed by three multiple-choice items (potential maximum score 3.0). Mean (SD) BT knowledge was $2.7(0.58)$ points. PFMT knowledge was assessed 2-4 weeks following group instruction by five multiple-choice items (potential maximum score 5.0). Mean (SD) PFMT knowledge was 4.3 (0.95) points. These results translate into a $90 \%$ level of correct knowledge of BT and an $86 \%$ correct knowledge of PFMT. Misunderstandings about BT and PFMT as indicated by incorrect responses to particular items were addressed during the clinical examination visit by the nurse specialist.

Recognizing the phased application of the inclusion/ exclusion criteria, 199 women provided initial clinical data on pelvic floor motor skill at 2-4 weeks following group education. Of this group 68\% (135/199) demonstrated effective pelvic muscle contraction technique and required no further instruction. Thirty-two percent $(64 / 199)$ did not demonstrate an effective contraction because they were missing at least one characteristic of correct pelvic muscle technique. They received further instruction and coaching. The digital measure scores to quantify baseline pelvic muscle strength were obtained after any necessary instruction. Women who were not able to generate any pressure or displacement following individualized coaching [ultimately 3\% (6/199)] were excluded from the study because we believed it was not reasonable to ask them to undertake a pelvic muscle and bladder training program if they were unable to contract the target muscles. To summarize the results of PFMT motor skill, $68 \%$ required no further instruction following the group instruction, $29 \%$ demonstrated effective technique after brief instruction, and 3\% were never able to contract the pelvic muscles.

Adherence was assessed at 3, 6, 9, and 12 months following BMP instruction. Women were queried about their practice of BT and PFMT. In the group session, 
participants who initially demonstrated a voiding interval of less than 3.5 or more than $4 \mathrm{~h}$ had been advised to actively implement BT. Of those who returned their baseline voiding diary $(159 / 164)$, a total of $79 \%(126 /$ 159) of participants had voiding intervals that were outside the recommended 3.5-4 h. Most were at shorter intervals, but a few were beyond the 4-h boundary. These individuals were candidates for active BT. At the 3 -month data point, $66 \%(105 / 159)$ of the individuals for whom BT was recommended had achieved the desired interval $( \pm 0.5 \mathrm{~h})$ or reported that they were practicing BT. See Table 4 for adherence reported at subsequent data points. At the 3-month time, women who should have been practicing BT but were not reported on barriers to BT practice. Reasons given for not practicing BT included: too complicated to fit into daily life $26 \%(7 / 27)$, not a technique that she wanted to try $4 \%(1 / 27)$, incorrect belief that she was within the recommended voiding interval $44 \%(12 / 27)$, and "other" $26 \%(7 / 27)$.

We recommended that PFMT be practiced every day by each participant. Reported adherence to PFMT is presented in Table 4. At the 3-month data point, $82 \%$ reported practicing PFMT at least 2-3 times a week. At the 12-month data point $68 \%$ reported practice of PFMT at least 2-3 times a week.

Due to the limitations of self-reported behaviors, we looked to the objective outcome measures from the RCT [3] to validate the BT and PFMT self-reports. Using data from 3-day voiding diaries at exit from the RCT after 12 months, voiding frequencies on three parameters ( $24 \mathrm{~h}$, while asleep, while awake) showed significant reductions in the treatment group $(p<0.0001)$. The voiding interval compared between baseline and exit also demonstrated significant improvement for the BMP treatment group $(p<0.0001)$. In real time, the voiding interval for the treatment group at 12 months compared to baseline increased by approximately $33 \mathrm{~min}$, i.e., from $3 \mathrm{~h} 8 \mathrm{~min}$ at baseline to $3 \mathrm{~h} 41 \mathrm{~min}$ at exit. Baseline pelvic floor muscle pressure and displacement scores for the treatment group and the control group were equivalent indicating effective randomization. At exit, paired differences for the pressure score and the displacement score demonstrated significance between groups. More participants in the treatment group, compared to their counterparts in the control group, showed improvement for the pressure and displacement differential $(p=0.0004$ and 0.0001 , respectively). Thus, significant increases in pelvic muscle pressure and displacement and lengthened voiding interval occurred from baseline to the 1-year exit point in the treatment group. These findings add to our confidence that, in fact, the women were using the BT and PFMT self-care strategies at the levels they selfreported.

\section{Discussion}

Self-report of adherence to the recommended PFMT protocols was moderate to high and compares favorably to adherence reported in other studies. For example, the 3-month self-report of adherence was $82 \%$ as compared to Morkved et al.'s [7] finding of $81 \%$ at 12 weeks post-intervention in a test of PFMT as a prevention strategy. Adherence to BT in this study attained levels of $60 \%$ or higher. To our knowledge this is the first report of BT adherence; hence, it was not possible to compare our BT adherence rates with other research reports.

African American women comprise 15\% (52/359) of our total sample, which according to current United States Census website data is comparable to African American representation in the State of Michigan $(14.2 \%)$ and in the total United States $(12.3 \%)$. This affords some generalizability to the general United States population, but further research should strive for increased diversity with respect to Latinas and other underrepresented racial/ethnic groups. Also, our sample was primarily healthy and well educated, factors associated with better adherence. However, given that healthier, better educated individuals are more likely to seek preventive care, our findings would be expected to replicate in a future community-based test of effectiveness.

This study is unique in that it reports knowledge, skill, and adherence to BT and PFMT over a period of 1 year. Our results demonstrate that essentially continent women learn and practice the self-care techniques of BT and PFMT as presented in the group session. The results add to the evidence that group instruction, with a brief individualized supplement as needed, is an effective teaching strategy for PFMT and demonstrate for the first time to our knowledge its effectiveness for BT. Following the group sessions and prior to any individ-

Table 4 Percentage adherence to BMP

\begin{tabular}{llllll}
\hline & & $\begin{array}{l}\text { 3-month } \\
\text { adherence }\end{array}$ & $\begin{array}{l}\text { 6-month } \\
\text { adherence }\end{array}$ & $\begin{array}{l}\text { 9-month } \\
\text { adherence }\end{array}$ & $\begin{array}{l}\text { 12-month } \\
\text { adherence }\end{array}$ \\
\hline \multirow{3}{*}{ PFMT } & $(n=164)^{\mathrm{a}}$ & $(\%)$ & $(\%)$ & $(\%)$ & $(\%)$ \\
& Low: 1 time per week & 18 & 25 & 37 & 32 \\
& Medium: $2-3$ times per week & 17 & 24 & 18 & 32 \\
BT & High: at least once a day & 65 & 51 & 45 & 36 \\
& Within or perceived themselves & 66 & 67 & 66 & 58 \\
& to be within recommended interval \\
& of $3.5-4.0$ h or actively practicing BT & & & & \\
\end{tabular}

${ }^{\mathrm{a} C}$ Cases reporting vary across time intervals 
ualized teaching, participants demonstrated very good levels of knowledge about BT $(90 \%)$ and PFMT $(86 \%)$ and nearly $70 \%$ were able to demonstrate effective pelvic floor muscle contraction technique, requiring no further instruction.

Although we did not obtain baseline levels of BT and PFMT knowledge prior to the group session, we are reasonably sure that participant knowledge was at a very general level because each denied previous or present UI treatment with behavioral techniques as a criterion for inclusion in the study. For example, participants might have been exposed to Kegels during the childbearing years, or perhaps to articles about BT or PFMT in popular magazines, but this information has historically been very general. Hence, we are confident that the specific knowledge about BT and PFMT and level of PFMT technique that our participants demonstrated did, in fact, result from the BMP group session.

Greater knowledge of the rationale and methods for BT and PFMT, in conjunction with effective motor skill for the performance of PFMT, will benefit the continence status of women [3]. Because sustaining the behavioral change is essential to the success of behavioral intervention, it is noteworthy that the BMP was effective in motivating a $68 \%$ PFMT adherence rate, i.e., practicing 2-3 times per week $(32 \%)$ or at least once a day $(36 \%)$ and a $58 \%$ BT (Table 4 ) adherence or within recommended interval at 12 months post-intervention.

Group instruction via a 2 -h session in conjunction with a brief individualized visit has the potential to be cost-effective with respect to provider time and clinical resources. A cost analysis was beyond the scope of this investigation, but is an important research question for subsequent study.

\section{Conclusions}

Based on the knowledge, motor skill, and long-term adherence outcomes of this investigation, we conclude that the BMP model of group teaching followed by brief (approximately $10 \mathrm{~min}$ ) individualized instruction effectively educates women about and motivates them to adopt and sustain the self-care strategies of BT and PFMT.

Acknowledgments The authors would like to acknowledge appreciation to Cindy Karl, BSN formerly of the Department of Urology Research at William Beaumont Hospital for her contributions and clinical data collection, and also our deep appreciation to A. Regula Herzog, M.A., Ph.D, for her scholarly contributions and whose untimely passing has left us with an irreplaceable loss (8 January 1941-28 July 2002). Grant support: funded by National Institutes of Health-National Institute of Aging MERIT Award R37 AGO8511 and Nursing R01 NR0718.

\section{References}

1. Fantl JA, Newman DK, Colling J et al (1996) Urinary incontinence in adults: acute \& chronic management. Clin Prac Guidel Quick Ref Guide Clin, No. 2, 1996 update (AHCPR Publication No. 96-0682). Rockville, MD, US Department of Health and Human Services, Public Health Service, Agency for Health Care Policy and Research

2. Holroyd-Leduc JM, Straus SE (2004) Management of urinary incontinence in women. JAMA 291:986-995

3. Diokno AC, Sampselle CM, Herzog AR et al (2004) Prevention of urinary incontinence by behavioral modification program: a randomized, controlled trial among older women in the community. J Urol 171:1165-1171

4. Dougherty MC, Dwyer JW, Pendergast JF et al (2002) A randomized trial of behavioral management for continence with older rural women. Res Nurs Health 25:3-13

5. Wyman JF, Fantl JA, McClish DK, Bump RC (1998) Comparative efficacy of behavioral interventions in the management of female urinary incontinence. Am J Obstet Gynecol 179:999-1007

6. Janssen CCM, Lagro-Janssen ALM, Felling AJA (2001) The effects of physiotherapy for female urinary incontinence: individual compared with group treatment. BJU Int 87:201-206

7. Morkved S, Bo K, Schei B et al (2003) Pelvic floor muscle training during pregnancy to prevent urinary incontinence: a single-blind randomized control trial. Obstet Gynecol 101:313319

8. Miller J, Ashton-Miller J, DeLancey J (1998) Quantification of cough-related urine loss in SUI using the paper towel test. Obstet Gynecol 91:705-709

9. Bandura A (1977) Self-efficacy: toward a unifying theory of behavioral change. Psychol Rev 84:191-215

10. van Ryn M, Vinokur AD (1992) How did it work? An examination of the mechanisms through which an intervention for the unemployed promoted job-search behavior. Am J Community Psychol 20:557-597

11. Vinokur AD, Schul Y (1997) Mastery and inoculation against setbacks as active ingredients in the JOBS intervention for the unemployed. J Consult Clin Psychol 65:867-877

12. Vinokur AD, Schul Y, Vuori J, Price RH (2000) Two years after a job loss: long-term impact of the JOBS program on reemployment and mental health. J Occup Health Psychol 5:32-47

13. Brink CA, Wells TJ, Sampselle CM, Taillie ER, Mayer R (1994) A digital test for pelvic muscle strength in women with urinary incontinence. Nurs Res 43:352-356

14. Peschers UM, Gingelmaier A, Jundt K, Leib B, Dimpfl T (2001) Evaluation of pelvic floor muscle strength using four different techniques. Int Urogynecol J Pelvic Floor Dysfunct $12: 27-30$

15. Wyman JF, Choi SC, Harkins SW, Wilson MS, Fantl JA (1988) The urinary diary in evaluation of incontinent women: a test-retest analysis. Obstet Gynecol 71:812-817

16. Morkved S, Bo K (1996) The effect of post-natal exercises to strengthen the pelvic floor muscles. Acta Obstet Gynecol Scand 75:382-385

17. Reilly ETC, Freeman RM, Waterfield MR, Waterfield AE, Steggles P, Pedlar F (2002) Prevention of postpartum stress incontinence in primigravidae with increased bladder neck mobility: a randomised controlled trial of antenatal pelvic floor exercises. BJOG 109:68-76

18. Sampselle CM, Miller JM, Mims BM, DeLancey JOL, AshtonMiller JA, Antonakos CL (1998) Effect of pelvic muscle exercise on transient incontinence during pregnancy and after birth. Obstet Gynecol 91:406-412 